\title{
Limitations of Gravimetric Quantitative Blood Loss during Cesarean Delivery
}

\author{
Robert L. Thurer, MD ${ }^{1}$ \\ Andrea T. Jelks, MD ${ }^{4}$ \\ ${ }^{1}$ Medical Division, Gauss Surgical, Inc., Menlo Park, California \\ 2 Department of Obstetrics and Gynecology, Santa Clara Valley \\ Medical Center, San Jose, California \\ ${ }^{3}$ Division of Obstetric Anesthesia, Department of Anesthesiology, \\ Perioperative and Pain Medicine, Stanford University School of \\ Medicine, Stanford, California \\ ${ }^{4}$ Division of Maternal Fetal Medicine, Department of Obstetrics and \\ Gynecology, Santa Clara Valley Medical Center, San Jose, California \\ AJP Rep 2022;12:e36-e40.
}

\section{Brendan Carvalho, MBBCh, FRCA, MDCH ${ }^{3}$}

\begin{abstract}
Address for correspondence Robert L. Thurer, MD, Gauss Surgical, Inc., 45 Province St., Apt. 2101, Boston, MA 02108 (e-mail: rthurer@gmail.com).
\end{abstract}

\begin{abstract}
Keywords

- cesarean delivery

- blood loss quantification

- postpartum hemorrhage

- quality improvement

Objective This study examined the accuracy, sources of error, and limitations of gravimetric quantification of blood loss (QBL) during cesarean delivery.

Study Design Blood loss determined by assays of the hemoglobin content on surgical sponges and in suction canisters was compared with QBL in 50 parturients.

Results QBL was moderately correlated to the actual blood loss $(r=0.564 ; p<0.001)$. Compared with the reference assay, QBL overestimated blood loss for 44 patients (88\%). QBL deviated from the assayed blood loss by more than $250 \mathrm{~mL}$ in 34 patients $(68 \%)$ and by more than $500 \mathrm{~mL}$ in 16 cases (32\%). Assayed blood loss was more than $1,000 \mathrm{~mL}$ in four patients. For three of these patients, $\mathrm{QBL}$ was more than $1,000 \mathrm{~mL}$ (sensitivity $=75 \%$ ). QBL was more than $1,000 \mathrm{~mL}$ in 12 patients. While three of these had an assayed blood loss of more than $1,000 \mathrm{~mL}, 9$ of the 46 patients with blood losses of less than $1,000 \mathrm{~mL}$ by the assay (20\%) were incorrectly identified as having postpartum hemorrhage by QBL (false positives). The specificity of quantitative QBL for detection of blood loss more than or equal to $1,000 \mathrm{~mL}$ was $80.4 \%$.

Conclusion QBL was only moderately correlated with the reference assay. While overestimation was more common than underestimation, both occurred. Moreover, QBL was particularly inaccurate when substantial bleeding occurred.
\end{abstract}

\section{Key Points}

- QBL is inaccurate in cesarean delivery.

- QBL deviated from the assay result by more than $500 \mathrm{~mL}$ in $32 \%$ of cases.

- QBL sensitivity and specificity for hemorrhage is $75.0 \%$ (95\% confidence interval [CI]: 0.19-0.93) and 80.4\% (95\% CI: 0.690.92), respectively.

received

June 16, 2020

accepted after revision

November 2, 2021
DOI https://doi.org/ $10.1055 / \mathrm{s}-0041-1742267$ ISSN 2157-6998.

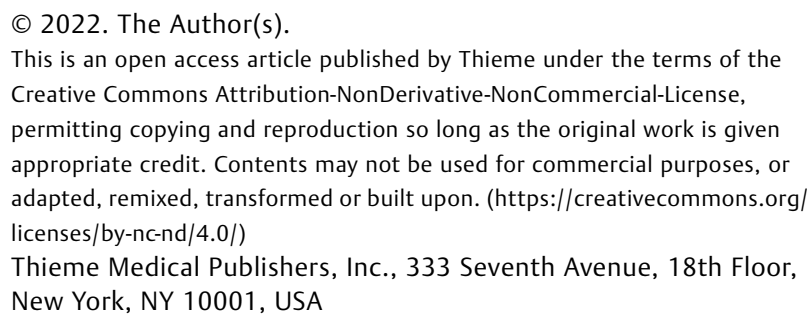
appropriate credit. Contents may not be used for commercial purposes, or adapted, remixed, transformed or built upon. (https://creativecommons.org/ licenses/by-nc-nd/4.0/) Thieme Medical Publishers, Inc., 333 Seventh Avenue, 18th Floor, New York, NY 10001, USA 
Quantification of blood loss (QBL) is recommended over visual estimation of blood loss for women having cesarean delivery. ${ }^{1,2} \mathrm{QBL}$ is thought to enhance the accuracy of blood loss measurements, improve hemorrhage recognition, and allow for appropriate activation of treatment stage-based protocols which may consequentially improve patient outcomes. $^{3}$

A common way of measuring $\mathrm{QBL}$ is the gravimetric method that involves weighing of soiled sponges and measurement of fluid in calibrated suction canisters. ${ }^{4}$ After adjusting for the dry weight of soiled materials, the estimated amount of amniotic fluid and the amount of surgical irrigation are deducted from the total weight, presumably resulting in an accurate measure of blood loss. While intuitively sensible, technical problems obtaining accurate measurements, difficulty in estimating amniotic fluid, and retention of irrigation fluid in the abdomen can lead to inaccurate results. ${ }^{5,6}$ Moreover, some blood loss may not be captured on surgical sponges and in suction canisters leading to additional error.

In a previous publication that compared visually estimated blood loss, gravimetric QBL, and a colorimetric method of estimating blood loss to a reference hemoglobin assay measurement of the amount of blood on surgical sponges and in suction canisters during scheduled cesarean delivery, we found that gravimetric QBL measurements were poorly correlated to the more accurate reference hemoglobin assay results. ${ }^{7}$ The aim of this secondary analysis was to determine the sources of error and limitations of the gravimetric QBL method in greater detail, using both correlations and BlandAltman measures of agreement.

\section{Materials and Methods}

The original protocol was approved by the Santa Clara Valley Medical Center Institutional Review Board (San Jose, CA; reference no.: 12-003; August 12, 2013). The details of the study methodology have been previously described. ${ }^{7}$ Briefly, all sponges and calibrated surgical canisters from 50 patients having scheduled cesarean deliveries were evaluated, and relevant patient and procedural information was collected. The original study compared visually estimated blood loss, gravimetric QBL, and a colorimetric blood loss estimation to a reference hemoglobin extraction assay during scheduled cesarean delivery. This secondary analysis is focused on the relationship between gravimetric QBL and the reference hemoglobin assay.

Quantitative gravimetric measurement methods were adopted from published guidelines. ${ }^{4}$ At the time of the uterine incision, the surgical technician or circulating nurse recorded the canister volume using the graduated markings. After aspiration of amniotic fluid, a second measurement was made, and the difference was recorded as the estimated amniotic fluid volume. At the conclusion of the surgery, the technician recorded the total amount of any irrigation fluid used. Additionally, any blood loss expressed by uterine massage was collected and transferred to one or more sponges. Immediately following the case, all sponges and suction canisters were individually weighed using a calibrated digital scale, and dry sponge weights and canister weights were subtracted. To determine the total QBL estimate, all individual sponge and canister measurements were tallied, and the measured amount of amniotic fluid and irrigation fluid used was subtracted from the total weight.

For the reference hemoglobin extraction assay method, all soiled laparotomy sponges and calibrated suction canisters were transferred to an on-site benchtop facility for hemoglobin measurement at the completion of each procedure. ${ }^{7}$ Sponges were individually soaked in $400 \mathrm{~mL}$ of normal saline, compressed by hand for 60 seconds to a mean weight of $50 \mathrm{~g}$. This process was repeated four times and the hemoglobin concentration of the final extraction fluid was measured using the plasma/low spectrophotometer (Hemo-Cue AB, Ängelholm, Sweden). The yield of the extraction process was independently characterized by depositing banked blood on sponges in known quantities and performing the same mechanical extraction. A linear regression analysis revealed mean hemoglobin recovery rates of $89.5 \%$ (95\% confidence interval [CI]: 86.8-92.1\%) for individual sponges ( $n=116)$ and this value along with the patient's preoperative hemoglobin was used to convert the extracted amount of hemoglobin $(\mathrm{g})$ to the amount of blood in the sponges $(\mathrm{mL})$.

The hemoglobin concentration in the canisters was separately assayed by using either a whole blood or low-concentration hemoglobin analyzer and converted to a canister blood volume based on the patient's preoperative hemoglobin concentration (in $\mathrm{g} / \mathrm{dL}$ ). ${ }^{7}$ The blood loss in the canisters was then combined with the blood loss from the sponges to give a total assayed blood loss. The blood and fluid that remained on the surgical drapes, gowns, towels, and other materials was not accounted for by either the quantitative gravimetric assessment or the hemoglobin assay.

\section{Statistical Analysis}

The sample size was based on the previous study. The distributions of the blood loss measurements and other analysis quantities were assessed using descriptive univariate statistics: the mean and standard deviation ( \pm ), as well as the median and the first and third quartiles. Since the primary outcome of this paper is the accuracy of the gravimetric method compared with the hemoglobin assay method (i.e., considered to be the accurate reference value), the absolute difference between the hemoglobin assay and gravimetric methods was assessed instead of the signed difference between the measures.

Estimates of sensitivity and specificity used a binomial model. Since only four cases were observed with assayed blood loss greater than $1,000 \mathrm{~mL}$, CIs for sensitivity were computed using the exact small-sample formula. CIs for specificity used the standard large sample formula for the binomial distribution.

The strength of the relationship between the gravimetric and assay methods was assessed by correlation coefficients; Fisher's $z$-transformation was used to compute CIs for correlation coefficients. A quantitative relationship between the gravimetric and assay methods was derived using the 
nonparametric regression method of Passing and Bablok. ${ }^{8}$ The Passing-Bablok regression line provides a nonparametric estimate of the linear relationship between two measures that assumes both are subject to error, without making further assumptions about the statistical distributions of the measures. The strength of correlations was assessed as follows: $r<0.5$ (low); $r=0.5$ to $<0.7$ (moderate), $r=0.7$ to $<0.9$ (high); and $r \geq 0.9$ (very high). For comparative analysis, a $p$-value of 0.05 was considered significant.

In addition, agreement between the extraction assay and gravimetric QBL was evaluated using the Bland-Altman method. ${ }^{9}$ The Bland-Altman bias (mean the difference between the two measures) and upper and lower limits of agreement (mean $\pm 1.96 \times \mathrm{SD}$ ) with the respective $95 \%$ CIs was computed. All statistical computations were computed using SAS, Version 9.4 (SAS Institute, Cary, NC).

\section{Results}

Data were successfully collected from all 50 patients. $^{7}$ The mean preoperative hemoglobin level was $12.2 \pm 1.0 \mathrm{~g} / \mathrm{dL}$. Additional patient characteristics were described in the previous publication. ${ }^{7}$

The gravimetric QBL method was moderately correlated to the actual (hemoglobin assayed) blood loss ( $r=0.564$; mean absolute difference $=422 \pm 330 \mathrm{~mL}$; median absolute difference $=365 ;[204-538] \mathrm{mL} ; p<0.001) .^{7}$ The $95 \% \mathrm{CI}$ for the correlation coefficient was [0.334, 0.725]. As previously reported, ${ }^{7}$ the assessment of agreement between gravimetric $\mathrm{QBL}$ and the extraction assay revealed a bias $(95 \% \mathrm{CI})$ of $353 \pm 405 \mathrm{~mL}$. The upper limit of agreement $(95 \% \mathrm{CI})$ was $1,145 \mathrm{~mL}$ and the lower limit of agreement (95\% CI) was $-441 \mathrm{~mL}$. The Bland-Altman plot is represented in - Fig. 1.

Compared with the hemoglobin assayed amount, gravimetric QBL overestimated blood loss for 44 of the 50 patients (88\%). In 32 patients (64\%) the overestimate was greater than $250 \mathrm{~mL}$ and for 14 patients (28\%), the overestimate was greater than $500 \mathrm{~mL}$. In two instances, there was an under-

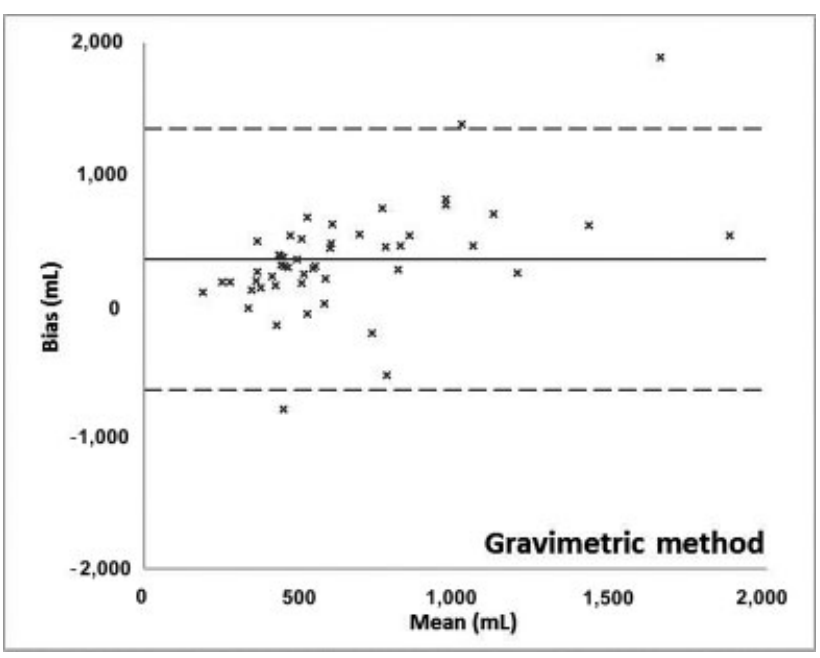

Fig. 1 Assessment of agreement between gravimetric QBL and the extraction assay method according to the Bland-Altman method. QBL, quantification of blood loss.

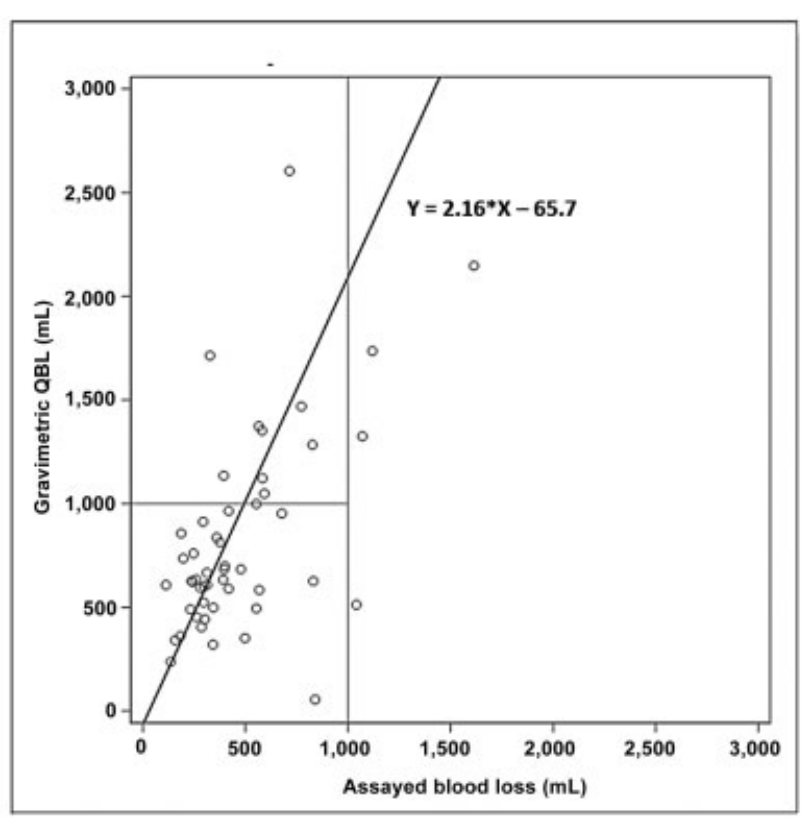

Fig. 2 Assayed blood loss versus gravimetric QBL with PassingBablock regression line. QBL, quantification of blood loss.

estimation of more than $500 \mathrm{~mL}$ when compared with the assay (528 and $784 \mathrm{~mL}$, respectively). Collectively, gravimetric QBL was only within $250 \mathrm{~mL}$ of the actual amount in 16 (32\%) patients and all but three of these patients had an assayed blood loss of less than $500 \mathrm{~mL}$ (mean $=378 \pm 179$ $\mathrm{mL}$ ). The Passing-Bablock regression line (-Fig. 2) demonstrates that gravimetric QBL is typically about twice the blood loss determined by the hemoglobin assay method.

Assayed blood loss was greater than $1,000 \mathrm{~mL}$ in four patients. For three of the four patients gravimetric QBL recognized that the blood loss was greater than $1,000 \mathrm{~mL}$ (sensitivity $=75 \%$; 95\% CI: $0.194-0.932$ ) but overestimated it by 617,533 and $255 \mathrm{~mL}$, respectively. For the fourth patient the assayed blood loss was $1,041 \mathrm{~mL}$ but the gravimetric QBL was only $513 \mathrm{~mL}$. Seventeen patients (34\%) had an assayed blood loss of greater than $500 \mathrm{~mL}$. For those 17 patients, the mean absolute difference between the QBL measurement and the assay was $549 \pm 420 \mathrm{~mL}$ (median $=528$; [275-696] $\mathrm{mL}$ ). Gravimetric QBL overestimated the assayed blood loss in 13 of the 17 patients (mean overestimation $=597 \pm 445$ $\mathrm{mL}$ ) and underestimated it in 4 (mean underestimation $=394 \pm 326 \mathrm{~mL}$ ). Only 8 of the 17 (47\%) values were within $500 \mathrm{~mL}$ and quantitative QBL was within $250 \mathrm{~mL}$ of the assayed result in only $3(18 \%)$ cases.

Gravimetric QBL was greater than $1,000 \mathrm{~mL}$ (the American College of Obstetricians and Gynecologists [ACOG] criteria for hemorrhage recognition ${ }^{10}$ ) in 12 patients. While three of these had an assayed blood loss of greater than $1,000 \mathrm{~mL}, 9$ of the 46 patients $(20 \%)$ with blood losses of less than $1,000 \mathrm{~mL}$ by the hemoglobin assay were incorrectly identified as having postpartum hemorrhage (false positives). The specificity of gravimetric QBL was 80.4\% (95\% CI: 0.690-0.919). In 37 cases (74\%), there was concordance among both methods that the blood loss was less than $1,000 \mathrm{~mL}$. These relationships are illustrated in -Table 1. Interestingly, if the criteria for hemorrhage 
Table 1 Distribution of blood loss by quantitative QBL and the reference assay $(\mathrm{mL})$

\begin{tabular}{|l|l|l|}
\hline & Assay $\leq 1,000 \mathrm{~mL}$ & Assay $>1,000 \mathrm{~mL}$ \\
\hline $\mathrm{QBL} \leq 1,000 \mathrm{~mL}$ & 37 & 1 \\
\hline $\mathrm{QBL}>1,000 \mathrm{~mL}$ & 9 & 3 \\
\hline
\end{tabular}

Abbreviation: QBL, quantification of blood loss.

recognition was $900 \mathrm{~mL}$ rather than $1,000 \mathrm{~mL}$, gravimetric QBL would have recognized hemorrhage in 16 patients, only 3 of whom had an assay of greater than $900 \mathrm{~mL}$. Thus, 13 of the 46 patients (28\%) with blood losses of less than $900 \mathrm{~mL}$ would have been incorrectly identified as having postpartum hemorrhage. Conversely, if the criteria for hemorrhage recognition were increased to $1,100 \mathrm{~mL}$, the false positive rate would be 8 of 47 patients (17\%).

Corrections for amniotic fluid and irrigation were collectively applied to the sponge and calibrated canister quantified amounts. The measured amount of amniotic fluid varied from 100 to $2,100 \mathrm{~mL}$ ( mean $=632 \pm 507 \mathrm{~mL}$; median $=500 \mathrm{~mL}$; [250-850]). The measured amount of irrigation varied from 50 to $2500 \mathrm{~mL}$ (mean $=760 \pm 437 \mathrm{~mL}$; median $=775 \mathrm{~mL}$; [400-1,000]).

An average of 15 surgical sponges were used per case ( mean $=15.1 \pm 4.9$; median $=14.5$; $[12-17])$. The gravimetric QBL on the sponges was substantially higher than the hemoglobin assayed amount of blood on the sponges in every case (mean absolute difference $=480 \pm 182 \mathrm{~mL}$ ). The number of sponges used was highly correlated with the total assayed blood loss on the sponges ( $r=0.748 ; 95 \% \mathrm{CI}$ : [0.588, 0.847]; $p<0.0001$; i.e., the use of more sponges was associated with a higher assayed blood loss).

A single-calibrated suction canister was utilized in 49 cases; the remaining case required use of two canisters. The assayed amount of blood in the canisters was substantially less than the total volume of fluid collected in the canisters

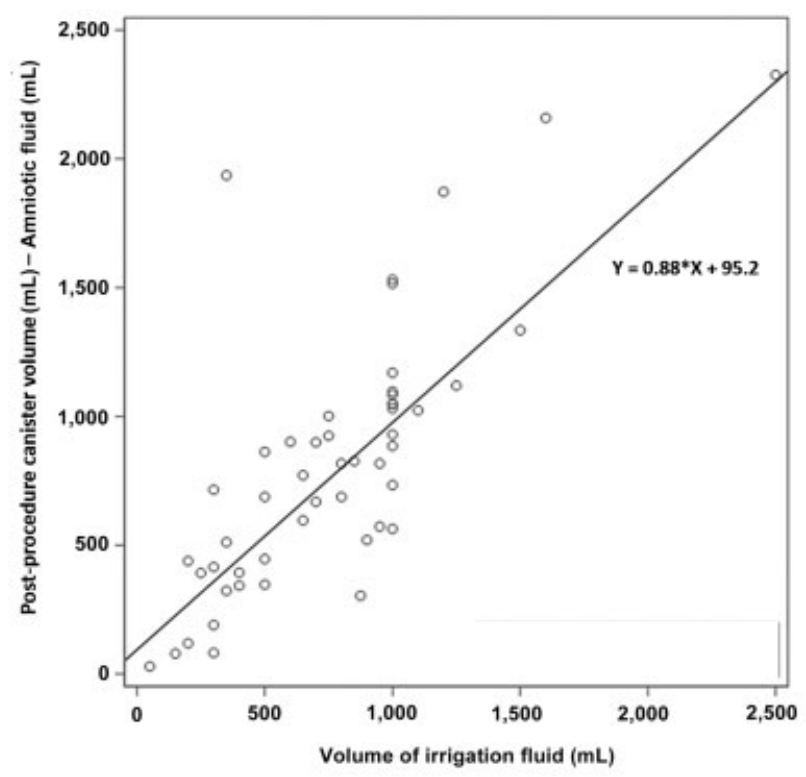

Fig. 3 Difference in canister volume (post-pre) versus irrigation volume $(\mathrm{mL})$ with Thiel regression line. (mean absolute difference $=1,264 \pm 627 \mathrm{~mL}$ ). The correlation between the total amount of fluid in the canisters and the actual amount of blood in the canister was low to moderate $\left(R^{2}=0.466 ; 95 \% \mathrm{CI}:[0.211,0.656]\right)$. Irrigation and amniotic fluids affected both the sponge and canister values. Assuming that all of the amniotic fluid was aspirated into the canisters, $88 \%$ of the irrigation fluid went into the canisters and only $12 \%$ was absorbed by the sponges (-Fig. 3 ).

\section{Discussion}

Our study found that despite the use of trained technicians to carefully perform gravimetric QBL using recommended guidelines, the results deviated substantially from the actual (hemoglobin assayed) amounts of blood on the soiled sponges and in the surgical canisters by greater than $250 \mathrm{~mL}$ in $68 \%$ of cases and by greater than $500 \mathrm{~mL}$ in $32 \%$ of cases. While overestimation was more common than underestimation, both occurred. Detection of hemorrhage (blood loss greater than $1,000 \mathrm{~mL})^{9}$ was aligned in 40 patients (80\%); however, in 10 cases (20\%), the results were not aligned. If quantitative $\mathrm{QBL}$ were used to implement a hemorrhage protocol, ${ }^{4}$ the protocol would have been inappropriately applied in the unaligned group.

Postpartum hemorrhage is the leading preventable cause of maternal mortality both in the United States and worldwide. ${ }^{11,12}$ Since treatment is often complicated by delayed or imprecise recognition, accurate and timely measurement of the actual amount of bleeding is recommended to identify hemorrhage early and institute protocol-based treatment plans that have led to improved outcomes. ${ }^{4,13}$

During vaginal delivery, blood loss is captured by calibrated under-buttocks drapes, surgical sponges and other substrates such as towels and pads that can be accurately weighed. Estimation of the volume of amniotic fluid is relatively simple since the collection of amniotic fluid precedes most of postpartum bleeding. During cesarean delivery, however, there is mixing of amniotic and irrigation fluid with maternal blood loss, further complicating the measurement of maternal blood loss.

\section{Strengths and Limitations}

The strength of our analysis is that the actual amount of blood loss was diligently assayed creating a blood loss "gold standard" for comparison. The major limitation was that only four of the patients studied ( $8 \%$ ) had a hemoglobin assayed blood loss of greater than $1,000 \mathrm{~mL}$, fulfilling the criteria for postpartum hemorrhage. ${ }^{13}$ The large differences in patients whose hemoglobin assayed blood loss was greater than $500 \mathrm{~mL}$ and greater than $1,000 \mathrm{~mL}$ demonstrate that the inaccuracy of quantitative $\mathrm{QBL}$ is substantial at higher amounts of blood loss.

Visual estimation of blood loss is known to be inaccurate. ${ }^{14,15}$ Therefore objective measures, such as gravimetric $\mathrm{QBL}$, are recommended and promoted. ${ }^{1,2}$ This analysis demonstrates significant inaccuracies in the gravimetric QBL method, suggesting alternative objective measures to 
accurately measure blood loss during cesarean delivery are needed. Several studies have shown that the colorimetric method is a practical and reliable way of accurately estimating maternal blood loss in the operating room. ${ }^{7,16}$ Using the change in hemoglobin from the preoperative level to the value following delivery, Saoud et al confirmed the accuracy of the colorimetric method. ${ }^{17}$ Katz et al recently demonstrated that quantifying blood loss using the colorimetric method resulted in improved identification of postpartum hemorrhage, changes in patient management and cost savings. ${ }^{18}$ Further studies regarding the utility of accurate QBL using the colorimetric method and its effect on reduction of maternal morbidity and mortality are needed.

\section{Conclusion}

In summary, this study demonstrates that quantitative QBL is only moderately correlated with actual blood loss and is potentially inaccurate when bleeding is substantial. These inaccuracies were apparent despite a dedicated assessor of gravimetric QBL and optimal methodology within a study. Further studies evaluating the quantitative method in patients with high-risk deliveries and in emergency cases with higher amounts of blood loss, as well as comparisons with alternative methodologies and effects on clinical outcomes, are needed.

\section{Funding}

Gauss Surgical, Inc. supplied statistical support for the study. Gauss personnel (R.L.T.) also participated in study design, data collection, data analysis, and article preparation.

\section{Conflict of Interest}

None declared.

\section{Acknowledgments}

The authors would like to recognize and thank our statistician William Krebs, PhD, and Griffeth Tully, MD, for reviewing the manuscript.

\section{References}

1 Quantification of blood loss: AWHONN practice brief number 1. J Obstet Gynecol Neonatal Nurs 2015;44(01):158-160

2 Quantitative blood loss in obstetric hemorrhage: ACOG COMMITTEE OPINION, number 794. Obstet Gynecol 2019;134(06): e150-e156
3 Main EK, Goffman D, Scavone BM, et al; National Partnership for Maternal Safety: Consensus Bundle on Obstetric Hemorrhage. Obstet Gynecol 2015;126(01):155-162

4 Lyndon A, Lagrew D, Shields LE, Main E, Cape V. Improving health care response to obstetric hemorrhage version 2.0. Accessed December 16, 2021: https://www.cmqcc.org/resource/obstetrichemorrhage-20-toolkit

5 Johar RS, Smith RP. Assessing gravimetric estimation of intraoperative blood loss. J Gynecol Surg 1993;9(03):151-154

6 Hamm RF, Wang E, Romanos A, O'Rourke K, Srinivas SK. Implementation of quantification of blood loss does not improve prediction of hemoglobin drop in deliveries with average blood loss. Am J Perinatol 2018;35(02):134-139

7 Doctorvaladan SV, Jelks AT, Hsieh EW, Thurer RL, Zakowski MI, Lagrew DC. Accuracy of blood loss measurement during cesarean delivery. AJP Rep 2017;7(02):e93-e100

8 Passing H, Bablok W. A new biometrical procedure for testing the equality of measurements from two different analytical methods. Application of linear regression procedures for method comparison studies in clinical chemistry, Part I. J Clin Chem Clin Biochem 1983;21(11):709-720

9 Bland JM, Altman DG. Statistical methods for assessing agreement between two methods of clinical measurement. Lancet 1986;1 (8476):307-310

10 Committee on Practice Bulletins-Obstetrics. Practice bulletin no. 183: postpartum hemorrhage. Obstet Gynecol 2017;130(04): e168-e186

11 Petersen EE, Davis NL, Goodman D, et al. Vital signs: pregnancyrelated deaths, United States, 2011-2015, and strategies for prevention, 13 states, 2013-2017. MMWR Morb Mortal Wkly Rep 2019;68(18):423-429

12 Say L, Chou D, Gemmill A, et al. Global causes of maternal death: a WHO systematic analysis. Lancet Glob Health 2014;2(06): e323-e333

13 Main EK, Cape V, Abreo A, et al. Reduction of severe maternal morbidity from hemorrhage using a state perinatal quality collaborative. Am J Obstet Gynecol 2017;216(03):298.e1-298.e11

14 Schorn MN. Measurement of blood loss: review of the literature.J Midwifery Womens Health 2010;55(01):20-27

15 Toledo P, Eosakul ST, Goetz K, Wong CA, Grobman WA. Decay in blood loss estimation skills after web-based didactic training. Simul Healthc 2012;7(01):18-21

16 Rubenstein AF, Zamudio S, Al-Khan A, et al. Clinical experience with the implementation of accurate measurement of blood loss during cesarean delivery: influences on hemorrhage recognition and allogeneic transfusion. Am J Perinatol 2018;35(07):655-659

17 Saoud F, Stone A, Nutter A, Hankins GD, Saade GR, Saad AF Validation of a new method to assess estimated blood loss in the obstetric population undergoing cesarean delivery. Am J Obstet Gynecol 2019;221(03):267.e1-267.e6

18 Katz D, Wang R, O'Neil L, et al. The association between the introduction of quantitative assessment of postpartum blood loss and institutional changes in clinical practice: an observational study. Int J Obstet Anesth 2020;42:4-10 